\title{
The Significance of Routine Biochemical Markers in Patients With Sepsis
}

\section{Yan Meng}

Tumor Hospital Affiliated to Xinjiang Medical University; First Affiliated Hospital of Xinjiang Medical University

Yue Wang

Tumor Hospital Affiliated to Xinjiang Medical University; First Affiliated Hospital of Xinjiang Medical University

Wenbin Qiao

Tumor Hospital Affiliated to Xinjiang Medical University

Yumei LIU

First Affiliated of Xinjiang Medical University

Liang Wang

First Affiliated Hospital of Xinjiang Medical University

Jiahui Fan

First Affiliated Hospital of Xinjiang Medical University

Fengming Tian

Tumor Hospital Affiliated to Xinjiang Medical University

Xuanzheng Wang

Tumor Hospital Affiliated to Xinjiang Medical University

Tao Zhang

First Affiliated Hospital of Xinjiang Medical University

Xiumin Ma ( $\square$ maxiumin1210@sohu.com )

Clinical Laboratory Center, Tumor Hospital Affiliated to Xinjiang Medical University https://orcid.org/0000-0001-8011-7513

\section{Research Article}

Keywords: conventional biomarkers, NE, WBC, D-Dimer, sepsis

Posted Date: December 31st, 2020

DOI: https://doi.org/10.21203/rs.3.rs-135063/v1

License: (9) This work is licensed under a Creative Commons Attribution 4.0 International License. Read Full License 


\section{Abstract}

Background: Sepsis is a highly complex and fatal syndrome. It is the main cause of death in the intensive care unit. Early diagnosis is beneficial to reduce the mortality of sepsis and improve the prognosis of patients. Therefore, we look forward to finding cheap and fast diagnostic criteria to quickly assess the patient's condition.

Methods: This is a retrospective study. The study enrolled 499 patients in the First Affiliated Hospital of Xinjiang Medical University from January 1, 2018 to June 22, 2020, and 96 healthy cases in the same period. Using the diagnostic criteria of bacterial infection, SIRS criteria and Sepsis- 2 consensus criteria, 499 patients and 96 healthy cases were divided into 4 groups: sepsis group $(n=300)$, SIRS group ( $n=151)$, infection group $(n=48)$, the control group $(n=96)$. We collected the results of routine laboratory tests, inflammation indicators and blood culture results of these patients.

Results: The sepsis group compared with the control group, MCV, NE, WBC, PLT, HB, D-Dimer, PT, CRP, PCT, IL-6, ALB, TBIL, Cr, LAC, CysC and BNP were statistically significant. D-dimer, CRP and PCT have higher diagnostic efficiency. Compared with the difference between the infection group and the SIRS group, PLT and IL- 6 are statistically significant, and have a certain diagnostic value. Sepsis group VS infection group, WBC, IL-6, NE and TBIL showed statistical differences in the comparison. The AUC of NE was 67.6, which was the largest among the three. The specificity (95.8\%) was the highest, but the sensitivity (49\%) was low.

Conclusions: This retrospective study shows that NE, WBC, and D-dimer can help in the early diagnosis of sepsis. D-dimer performs best. WBC and NE may have a differential diagnosis significance between the sepsis group and the infection group. This result can provide a timely and convenient assessment tool for early diagnosis of sepsis.

\section{Introduction}

Sepsis is a life-threatening organ dysfunction caused by the host's dysregulated response to infection(1,2). In 2017, 48.9 million cases of sepsis and 11 million deaths from sepsis occurred. The high mortality rate has caused extensive clinical concern for sepsis $(3,4)$.

Sepsis occurs on the basis of infection, involves systemic inflammatory response to infection, and manifests as microvascular dysfunction, coagulation disorders, and ultimately lead to multiple organ dysfunction $(5,6)$. The clinical manifestations of sepsis are easily interfered by many factors in the early stage, and lack of specificity, making its clinical manifestations often misleading and difficult to diagnose. Cultivation of pathogenic bacteria from blood or body fluids is regarded as the "gold standard" for diagnosing infection(7). However, it takes a long time to detect (mostly 3 to 5 days) and is susceptible to many factors, which can cause delays in diagnosis and treatment. Studies have suggested that the delay in the first use of antibiotics is related to the increase in hospital mortality, and early use of antibiotics requires early identification of sepsis and finding effective early diagnosis indicators, which is conducive to early diagnosis and early treatment of sepsis patients and reduces patient deaths rate( 8,9$)$.

Although sepsis3.0 diagnostic criteria have been proposed, there are still controversies regarding it. Sepsis3.0 diagnostic criteria have higher specificity, while Sepsis2.0 diagnostic criteria have better sensitivity(10). Sepsis3.0 focuses on organ dysfunction. Most of the patients who died of sepsis in the clinic are patients with early sepsis who have not yet shown organ dysfunction. The application of sepsis3.0 diagnostic criteria is not conducive to early identification of sepsis. Studies have suggested that the Sepsis2.0 diagnostic criteria can predict the adverse consequences of sepsis in emergency patients, and speculate that those patients with sepsis who can benefit most from early treatment. In addition, the sepsis 3.0 diagnostic criteria uses the SOFA score, which is not widely used in clinical practice. This study is a retrospective study and it is impossible to collect relevant data retrospectively. Therefore, the use of the Sepsis2.0 diagnostic criteria is more practical and beneficial to early diagnosis and treatment $(11,12)$.

This article is a retrospective study that evaluated the diagnostic value of infection and coagulation biomarkers in early laboratory test results. We analyze the diagnostic value of NE, WBC, D-dimer and other indicators. This study aims to clarify the applicability of early inflammation and coagulation routine laboratory test results as an early diagnosis of sepsis. Contribute to early diagnosis and early treatment and reduce patient mortality.

\section{Materials And Methods}

This study collected general information and the first auxiliary inspection results of 499 patients who visited the First Affiliated Hospital of Xinjiang Medical University from January 1, 2018 to June 22, 2020, and 96 healthy individuals who visited the hospital during the same period. Among them, 499 patients all had blood culture results. We applied Sepsis2.0 diagnostic criteria: sepsis was defined as a proven bacterial infection and systemic inflammatory response syndrome (SIRS). SIRS diagnostic criteria: must meet at least two of the following criteria: body temperature $\geq 38^{\circ} \mathrm{C}$ or $\leq 36^{\circ} \mathrm{C}$, heart rate $\geq 90$ beats per minute, tachypnea (respiratory rate $\geq 20 /$ min or hyperventilation: PaCO2 $\leq$ 
$32 \mathrm{mmHg}$ )and leukocytosis ( $\geq 12,000 / \mathrm{cu} \mathrm{mm}$ ) or leukopenia( $\leq 4,000 / \mathrm{cu} \mathrm{mm}){ }^{(13)}$. Infection diagnostic criteria: when the same strain is detected more than twice in the blood culture results, the patient is considered to have infection. According to the bacterial infection standard, SIRS diagnostic standard and sepsis diagnostic standard, 499 patients were divided into 3 groups, namely, sepsis group (infection plus SIRS), SIRS group (two or more SIRS criteria), infection group (positive blood culture with zero or one SIRS criterion). In addition to the healthy controls, there are four groups $(14,15)$.

\section{Exclusion Criteria}

(1) The subjects were under 18 years old or over 80 years old; (2) Have blood system diseases or immunodeficiency diseases; (3) Hcave received blood transfusion in the past 4 months, and/or have received blood transfusion in the past 14 days. (4) Patients with platelet transfusion or platelet count <20000/ $\mu \mathrm{L}$; (5) Patients take hormones; (6) Mental illness patients; (7) Pregnant women or tumor patients.

\section{Determination Of Biomarkers}

Blood samples were collected using EDTA-K2 anticoagulation vacuum tubes (Becton, Dickinson and Company, New Jersey, USA), and then MO, MVC, NE, WBC, PLT, HB (SYSMEX XN-2000, Japan) were detected within 30 minutes. Blood samples were collected using sodium citrate anticoagulation vacuum tubes (Becton, Dickinson and Company, New Jersey, USA) and centrifuged at 3000 rpm for 10 minutes, and then DDimer, PT (Werfen, ACLTOP 300, USA) was detected within 30 minutes. Blood samples were collected using lithium heparin anticoagulation tubes (Becton, Dickinson and Company, New Jersey, USA) and centrifuged at 3000 rpm for 10 minutes, and then ALB, TBIL, Cr, LAC, CysC (Roche cobas 700, Roche Diagnostics, Mannheim, Germany), CRP, PCT (Roche cobas 700, Roche Diagnostics, Mannheim, Germany), IL-6 (ADVIA Chemistry XPT System, Germany), BNP (Ortho Clinical Diagnostics, China).

\section{Statistical Analysis}

SPSS 26.0 software (BMI, Chicago, USA) was used to analyze the data. Qualitative and quantitative variables are expressed as count and percentage or mean \pm standard deviation. Difference analysis: Kruskal-Wallis test was used to compare the differences between the sepsis group and the other groups. Use receiver operating characteristic curve (ROC) to obtain the best cutoff value with Youden index to establish the cutoff point, and evaluate the diagnostic ability by calculating the area under the curve (AUC), sensitivity and specificity. Analyze the diagnostic value of NE, WBC, D-dimer and other indicators for sepsis. Statistical significance level was set as $P<0.05$.

\section{Results}

\section{Clinical data of patients}

A total of 596 patients are enrolled into the study,There are 300 cases (50.4\%) in the sepsis group, 151 cases (25.4\%) in the SIRS group, 48 cases (8.1\%) in the infection group, and 96 cases (16.1\%) in the control group. The clinical data of patients were shown in Table 1 and bacteriological findings were shown in Table 2. Kruskal-Wallis test was used for the difference analysis: Neutrophil count (NE), white blood cell count (WBC), interleukin-6 (IL-6) and total bilirubin (TBIL) were significantly different between the sepsis group and the SIRS group (P<0.05). There were statistically significant differences in gender, age, MCV, NE, WBC, PLT, HB, D-Dimer, PT, CRP, PCT, IL-6, ALB, TBIL, Cr, BNP, LAC and CysC between the sepsis group and the control group $(P<0.05)$. 
Table 1

baseline characteristics, Inflammatory marker

\begin{tabular}{|c|c|c|c|c|c|c|c|}
\hline Characteristic & Sepsis & SIRS & Infection & Control & $P$ value & & \\
\hline & $\mathrm{n}=300$ & $n=151$ & $\mathrm{n}=48$ & $\mathrm{n}=96$ & $\begin{array}{l}\text { Sepsis } \\
\text { vs } \\
\text { SIRS }\end{array}$ & $\begin{array}{l}\text { Sepsis } \\
\text { vs } \\
\text { Infection }\end{array}$ & $\begin{array}{l}\text { Sepsis } \\
\text { vs } \\
\text { Control }\end{array}$ \\
\hline $\operatorname{Sex}(m / f)$ & $187 / 113$ & $90 / 61$ & $27 / 21$ & $37 / 59$ & NS & NS & NS \\
\hline Death,n & 50 & 25 & 9 & 0 & NS & NS & 0.000 \\
\hline Age (year) & $60[47,74]$ & $55 \pm 17$ & $67[55,75]$ & $48 \pm 15$ & 0.008 & NS & 0.000 \\
\hline $\operatorname{MCV}(f l)$ & $89.8[85.73,93.58]$ & $89.30[85.48,92.98]$ & $90.05[86.3,95]$ & $91.85[88.13,94.3]$ & NS & NS & 0.016 \\
\hline $\mathrm{MO}\left(\times 10^{9} / \mathrm{L}\right)$ & $0.52[0.25,0.84]$ & $0.05[0.24,0.88]$ & $0.42[0.17,0.81]$ & $0.46 \pm 0.13$ & NS & NS & NS \\
\hline $\operatorname{NE}\left(\times 10^{9} / \mathrm{L}\right)$ & $10.02[5.7,16.15]$ & $10.11[4.88,15.99]$ & $7.34[5.23,8.83]$ & $3.62 \pm 1.06$ & NS & 0.000 & 0.000 \\
\hline $\begin{array}{l}\text { WBC (x } \\
\left.10^{9} / L\right)\end{array}$ & $12.17[7.26,17.85]$ & $11.3[6.3,18.15]$ & $8.7[6.60,11]$ & $6.38 \pm 1.51$ & NS & 0.000 & 0.000 \\
\hline $\operatorname{PLT}\left(\times 10^{9} / \mathrm{L}\right)$ & $158[82.25,260.75]$ & $110[66,225]$ & $147[107.25,232.75]$ & $238.94 \pm 51.71$ & 0.011 & NS & 0.000 \\
\hline $\mathrm{HB}(\mathrm{g} / \mathrm{L})$ & $110.36 \pm 29.25$ & $103.41 \pm 26.7$ & $103.41 \pm 26.7$ & $140.5 \pm 14.14$ & NS & NS & 0.000 \\
\hline $\begin{array}{l}\text { D-Dimer } \\
(\mu \mathrm{g} / \mathrm{L})\end{array}$ & $1385.5[784,3445]$ & $1760[796,3833]$ & $1002[518,2880]$ & $74.5[46.75,129]$ & NS & NS & 0.000 \\
\hline PT (s) & $14.7[12.9,16.3]$ & $14.5[12.53,18.3]$ & $14.15[12.35,15.95]$ & $11.32 \pm 0.84$ & NS & NS & 0.000 \\
\hline $\begin{array}{l}\text { CRP } \\
(\mathrm{mg} / \mathrm{mL})\end{array}$ & $90[56.3,176.93]$ & $90[36.7,170.37]$ & $90[33.34,168.88]$ & $5[5,5.08]$ & NS & NS & 0.000 \\
\hline PCT (ng/mL) & $4.45[0.72,33.31]$ & $5.85[1.31,27.52]$ & $1.42[0.25,31.14]$ & $0.03[0.02,0.0 .04]$ & NS & NS & 0.000 \\
\hline IL-6 (pg / mL) & $202.1[58.83,784.75]$ & $132.5[41.31,571.4]$ & $112.8[28.05,364.98]$ & $2.5[1.5,3.97]$ & 0.046 & 0.021 & 0.000 \\
\hline $\operatorname{ALB}(g / L)$ & $31.02[26.44,36.3]$ & $31.4[26.47,35.01]$ & $32.09 \pm 6.97$ & $44.29 \pm 3.49$ & NS & NS & 0.000 \\
\hline $\begin{array}{l}\text { TBIL } \\
(\mu \mathrm{mol} / \mathrm{L})\end{array}$ & $21.31[14.10,45.94]$ & $23.27[13.15,59.5]$ & $17.27[10.63,28.89]$ & $10.25[7.7,14.85]$ & NS & 0.026 & 0.000 \\
\hline $\mathrm{Cr}(\mu \mathrm{mol} / \mathrm{L})$ & $96.03[68.26,178.69]$ & $93.19[57.23,207]$ & $90.51[64.08,198.36]$ & $62.05[55,73.94]$ & NS & NS & 0.000 \\
\hline $\mathrm{BNP}(\mathrm{pg} / \mathrm{ml})$ & $2.41[1.66,4.07]$ & $659[153,3028.38]$ & $2.46[1.58,3.19]$ & $30.35[18.51,53.5]$ & NS & NS & 0.000 \\
\hline $\begin{array}{l}\mathrm{LAC} \\
(\mathrm{mmol} / \mathrm{L})\end{array}$ & $1.28[0.89,1.95]$ & $2.84[1.85,4]$ & $1.38[0.8975,2.33]$ & $2.2[1.7,2.66]$ & NS & NS & 0.019 \\
\hline CysC (mg/L) & $677.25[185.46,2662.5]$ & $1.38[0.93,2.24]$ & $876.83[149.65,3040]$ & $0.78[0.63,0.92]$ & NS & NS & 0.000 \\
\hline
\end{tabular}

\section{Blood Culture Data}

After analyzing the biomarkers of infection and blood coagulation in the patients' early laboratory test results, we checked their microbial blood culture results. The results of microbial blood culture in the sepsis group are shown in Table 2. Among them, 283 patients had mixed infections. Table 3 shows the microbial blood culture results of the infection group, and 16 patients had mixed infections. 
Table 2

Blood culture data for the cases in Sepsis group

\begin{tabular}{|c|c|c|c|}
\hline Sepsis group & Pathogen species & Number of cases & Percentage of total(\%) \\
\hline \multirow[t]{8}{*}{ Gram-negative bacteria $(\mathrm{n}=309)$} & Escherichia coli & 87 & 28.16 \\
\hline & Acinetobacter baumannii & 72 & 23.30 \\
\hline & klebsiella pneumoniae & 71 & 22.98 \\
\hline & Stenotrophomonas maltophilia & 16 & 5.18 \\
\hline & Pseudomonas aeruginosa & 18 & 5.83 \\
\hline & Klebsiella cepacia & 8 & 2.59 \\
\hline & Aerobacter cloacae & 10 & 3.24 \\
\hline & Others & 27 & 8.74 \\
\hline \multirow[t]{7}{*}{ Gram-positive bacteria $(n=154)$} & Enterococcus faecium group D & 76 & 49.35 \\
\hline & Staphylococcus hominis & 16 & 10.39 \\
\hline & Streptococcus viridis & 13 & 8.44 \\
\hline & Staphylococcus aureu & 22 & 14.29 \\
\hline & Streptococcus angina & 6 & 3.90 \\
\hline & Enterococcus faecalis group $D$ & 9 & 5.84 \\
\hline & Others & 12 & 7.79 \\
\hline \multirow[t]{5}{*}{ Fungus(n = 139) } & Moniliaalbican & 62 & 44.60 \\
\hline & Candida krusei & 27 & 19.42 \\
\hline & Candida tropicalis & 13 & 9.35 \\
\hline & Candida glabrata & 33 & 23.74 \\
\hline & Others & 4 & 2.88 \\
\hline Acid-fast bacterium(n = 1) & Mycobacterium tuberculosis & 1 & 1.00 \\
\hline \multicolumn{4}{|c|}{$\begin{array}{l}\text { *Others:The number of bacteria is less than } 6 \text { (Gram-negative bacteria:Aeromonas hydrophila,Sphingomonaspaucimobilis, Klebsiella } \\
\text { ornithiolyticus,Providencia rettgeri,Morganella morganii,Salmonella typhosa,Klebsiella oxytoca,Baeteroides fragilis,Enterobacter } \\
\text { aerogen,Proteus mirabilis,Citrobacter freundii,Serratia marcescens.Gram-positive bacteria:Enterococcus,Streptococcus equi } \\
\text {,Mlicrococcusscarlatinae,Enterococcus casseliflavus,Streptococcus constellatus,Streptococcus agalactiae,Enterococcus gallinarum } \\
\text { D,Streptococcus salivarius,Streptococcus mitis.Fungus:Candida guilliermondii,Saccharomyces cerevisiae,Candida lusitaniae,Streptomyces } \\
\text { raffinose). }\end{array}$} \\
\hline
\end{tabular}


Table 3

Blood culture data for the cases in Infection group

\begin{tabular}{|c|c|c|c|}
\hline Infection group & Pathogen species & Number of cases & Percentage of total(\%) \\
\hline \multirow[t]{4}{*}{ Gram-negative bacteria $(\mathrm{n}=50)$} & Escherichia coli & 19 & 38.00 \\
\hline & Acinetobacter baumannii & 5 & 10.00 \\
\hline & klebsiella pneumoniae & 14 & 28.00 \\
\hline & Others & 12 & 24.00 \\
\hline \multirow[t]{3}{*}{ Gram-positive bacteria $(n=14)$} & Enterococcus faecium group $D$ & 5 & 35.71 \\
\hline & Streptococcus viridis & 4 & 28.57 \\
\hline & Others & 5 & 35.71 \\
\hline \multirow[t]{4}{*}{ Fungus $(n=12)$} & Moniliaalbican & 4 & 33.33 \\
\hline & Candida krusei & 4 & 33.33 \\
\hline & Candida tropicalis & 1 & 8.33 \\
\hline & Candida glabrata & 3 & 25.00 \\
\hline
\end{tabular}

Sepsis group vs Control group The diagnostic value of patient laboratory examination results in patients with sepsis

Table 4 lists Cutoff values, sensitivity, specificity, 95\% confidence interval, and AUC for laboratory examination results. The Cutoff value is taken from the ROC curve (Fig. 2). The area under the horizontal curve (AUC) of D-dimer, CRP, PCT was significantly larger than other indexes(Ddimer: $A U C=99.3 \%, 95 \% C l: 0.976-1.000$, CRP: $A U C=99.8 \%, 95 \% C l: 0.990-1.000$, PCT: AUC = 99.9\%,95\%Cl: 0.994-1.000,P<0.05). The sensitivities of D-dimer, CRP and PCT were high(97.4\%), diagnostic performances are superior to other indicators. Their Cutoff values are respectively $300 \mu \mathrm{g} / \mathrm{L}, 12.695 \mathrm{mg} / \mathrm{mL}$ and $0.080 \mathrm{ng} / \mathrm{mL}$. 
Table 4

Evaluation of biomarkers in indicating sepsis by ROC (Sepsis vs Control)

\begin{tabular}{|c|c|c|c|c|c|c|}
\hline Biomarkers & AUC (\%) & Sensitivity (\%) & Specificity (\%) & Cutoff value & $95 \% \mathrm{Cl}$ & P Value \\
\hline MCV (fl) & 41.8 & 12.8 & 100.0 & 99.1 & $0.248-0.589$ & $P>0.05$ \\
\hline $\mathrm{NE}\left(\times 10^{9} / \mathrm{L}\right)$ & 86.5 & 79.5 & 100.0 & 5.5 & $0.765-0.965$ & $P<0.05$ \\
\hline WBC $\left(\times 10^{9} / \mathrm{L}\right)$ & 77.9 & 66.7 & 100.0 & 9.3 & $0.655-0.902$ & $P<0.05$ \\
\hline $\mathrm{HB}(\mathrm{g} / \mathrm{L})$ & 10.6 & 5.1 & 100.0 & 176.0 & $0.010-0.202$ & $P<0.05$ \\
\hline $\operatorname{PLT}\left(\times 10^{9} / \mathrm{L}\right)$ & 35.9 & 28.2 & 90.9 & 335.0 & $0.213-0.504$ & $P>0.05$ \\
\hline D-Dimer $(\mu \mathrm{g} / \mathrm{L})$ & 99.3 & 97.4 & 100.0 & 300.0 & $0.976-1.000$ & $P<0.05$ \\
\hline PT (s) & 93.9 & 92.3 & 100.0 & 12.0 & $0.870-1.000$ & $P<0.05$ \\
\hline CRP $(\mathrm{mg} / \mathrm{mL})$ & 99.8 & 97.4 & 100.0 & 12.7 & $0.990-1.000$ & $P<0.05$ \\
\hline PCT (ng/mL) & 99.9 & 97.4 & 100.0 & 0.1 & 0.994-1.000 & $P<0.05$ \\
\hline IL-6 (pg / mL) & 98.1 & 97.4 & 100.0 & 8.9 & $0.944-1.000$ & $P<0.05$ \\
\hline $\mathrm{BNP}(\mathrm{pg} / \mathrm{ml})$ & 97.4 & 89.7 & 100.0 & 126.5 & $0.938-1.000$ & $P<0.05$ \\
\hline $\operatorname{ALB}(g / L)$ & 0.1 & 0.0 & 100.0 & 50.9 & $0.000-0.028$ & $P<0.05$ \\
\hline $\mathrm{Cr}(\mu \mathrm{mol} / \mathrm{L})$ & 59.9 & 56.4 & 27.3 & 81.0 & $0.424-0.774$ & $P>0.05$ \\
\hline CysC (mg/L) & 74.7 & 69.2 & 81.8 & 1.0 & $0.607-0.887$ & $P<0.05$ \\
\hline LAC (mmol/L) & 54.0 & 20.5 & 0.0 & 3.6 & $0.357-0.722$ & $P>0.05$ \\
\hline TBIL $(\mu \mathrm{mol} / \mathrm{L})$ & 88.6 & 79.5 & 81.8 & 13.2 & $0.781-0.990$ & $P<0.05$ \\
\hline
\end{tabular}

Sepsis group vs SIRS group The differential diagnostic value of PLT and IL-6 in patients with sepsis and SIRS

K-w difference analysis indicated that PLT and IL- 6 had statistical significance in patients with sepsis and SIRS (P< 0.05$)$. Table 5 lists the cutoff values of PLT, IL-6, sensitivity, specificity, $95 \%$ confidence interval, and AUC. Sensitivity and specificity of the studied parameter, and the Cutoff value is calculated at the specified Cutoff value, which is taken from the ROC curve (Fig. 3). Although, IL-6(AUC = 55.7\%,95\%Cl: $0.501-$ $0.614, \mathrm{P}<0.05)$ and PLT(AUC $=57.3 \%, 95 \% \mathrm{Cl}$ : 0.518-0.629) has differential diagnostic significance in patients with sepsis and SIRS, its diagnostic efficacy is general.

Table 5

Evaluation of biomarkers in indicating sepsis by ROC (Sepsis vs SIRS)

\begin{tabular}{|llllll|}
\hline Biomarkers & AUC (\%) & Sensitivity (\%) & Specificity (\%) & Cutoff value & $95 \% \mathrm{Cl}$ \\
\hline IL-6 $(\mathrm{pg} / \mathrm{mL})$ & 55.7 & 60.7 & 50.3 & 132.7 & $0.501-0.614$ \\
PLT $\left(\times 10^{9} / \mathrm{L}\right)$ & 57.3 & 59.3 & 57.0 & 124.5 & $0.518-0.629$ \\
*AUC, area under the curve; NPV, negative predictive value; PPV, positive predictive value.
\end{tabular}

Sepsis group vs Infection group The differential diagnostic value of NE, WBC, IL-6 and TBIL in patients with sepsis and infection

Table 6 lists cutoff values of NE, WBC, IL-6, TBIL, sensitivity, specificity, 95\% confidence interval, and AUC. The sensitivity and specificity of the studied parameter are calculated by the specified Cutoff value, which is taken from the ROC curve (Fig. 4). When the cutoff point of NE greater than 10.5 was selected, the sensitivity and specificity were $49 \%$ and $95.8 \%$, respectively. The sensitivity and specificity of the diagnosis of sepsis were $51 \%$ and $89.6 \%$, respectively, when the cutoff point of WBC greater than 12 was selected. When the cutoff point of IL- $6>84.7$ was selected, the sensitivity and specificity of sepsis diagnosis were $70 \%$ and $47.9 \%$, respectively. When the cutoff point with TBIL greater than 20.2 was selected, the sensitivity and specificity for the diagnosis of sepsis were $55.3 \%$ and $64.6 \%$, respectively. Area under the NE curve (AUC) was greater than WBC, IL-6 and TBIL (AUC $=67.6 \%, 95 \% \mathrm{Cl}: 0.613-0.738, \mathrm{P}<0.05)$, and its specificity $(95.8 \%)$ was better than WBC, IL-6 and TBIL. The sensitivity (70\%) of IL- 6 was higher than that of NE, WBC and TBIL, and the area (AUC) under ROC curve was 60.4 (AUC $=60.4 \%, 95 \%$ Cl: $0.514-0.693)$, and the Cutoff value was $84.7 \mathrm{pg} / \mathrm{mL}$. 
Table 6

Evaluation of biomarkers in indicating sepsis by ROC (Sepsis vs Infection)

\begin{tabular}{|llllll|}
\hline Biomarkers & AUC (\%) & Sensitivity (\%) & Specificity (\%) & Cutoff value & $95 \% \mathrm{Cl}$ \\
\hline NE $\left(\times 10^{9} / \mathrm{L}\right)$ & 67.6 & 49.0 & 95.8 & 10.5 & $0.613-0.738$ \\
\hline WBC $\left(\times 10^{9} / \mathrm{L}\right)$ & 66.7 & 51.0 & 89.6 & 12.0 & $0.600-0.734$ \\
\hline IL-6 $(\mathrm{pg} / \mathrm{mL})$ & 60.4 & 70.0 & 47.9 & 84.7 & $0.514-0.693$ \\
\hline TBIL $(\mu \mathrm{mol} / \mathrm{L})$ & 60.0 & 55.3 & 64.6 & 20.2 & $0.511-0.689$ \\
\hline *AUC, area under receiver operating caracteristic curve; Cl, confidence interval. & \\
\hline
\end{tabular}

\section{Discussion}

Sepsis refers to the disorder of the body's response to infection and causes organ dysfunction that can threaten the body(16),Sepsis affects almost every organ system. The management of sepsis depends on early recognition and empirical antimicrobial treatment, fluid resuscitation and vasopressin treatment $(17,18)$. At present, there is no gold standard for diagnosis of sepsis, which is extremely challenging for its diagnosis and is one of the most important causes of death in the world. More than 30 million people are diagnosed with sepsis each year, and 5 million of them die. Even if the condition is relieved, many patients have long-term sequelae, which requires long-term care and brings a great burden to society and families. The World Health Assembly and WHO in 2017 make sepsis a global health priority(19). Although the global trend of sepsis reduction has been shown, it is important that there are still huge differences in the total number of sepsis between regions. Early diagnosis and early treatment are meaningful for improving patient prognosis and reducing mortality. Therefore, we hope to find simple and cheap diagnostic criteria(15,19).

In this study, 499 patients and 96 healthy individuals were included, and were divided into sepsis group $(n=300)$, SIRS group ( $n=151)$, infection group $(n=48)$, and control group $(n=96)$ using diagnostic criteria for bacterial infection, systemic inflammatory response syndrome and Sepsis2.0 diagnostic criteria. Kruskal-Wallis test was used to compare NE, WBC, D-Dimer, PT, CRP, PCT and IL-6, and ROC curve was drawn to evaluate the diagnostic efficacy of parameters.

PCT, IL-6, and CRP have always played an important role in the early diagnosis, disease evaluation and prognosis judgment of sepsis(20,21). However, CRP and PCT are both inconsistent in their diagnostic capabilities(22). CRP has been widely studied for sepsis. Its diagnostic accuracy is called into question because the results are inconsistent and variable depending on the severity of the disease and infection (15). One study suggested that CRP was less sensitive (AUC81, 96\% sensitivity, 79\% specificity) and less diagnostic value than PCT in sepsis (23). This is consistent with the results of our study, in which PCT was superior to CRP in the diagnosis of sepsis (AUC99.9, sensitivity $97.4 \%$, specificity $100 \%$ ) compared with healthy controls, which was beneficial to the diagnosis of sepsis. However, there was no statistical significance in the identification of sepsis in the SIRS group and the infection group $(P>0.05)$. Studies of its diagnostic value have yielded mixed results, a meta-analysis found that the AUC of PCT was 0.85 , the sensitivity and specificity of predicting sepsis in critically ill patients were $77 \%$ and $79 \%(24)$. A retrospective study found that the diagnostic utility of PCT in predicting sepsis was relatively low (15). PCT is clinically used to distinguish infectious sepsis from non-infectious diseases, which is consistent with our study. PCT levels were significantly higher in the sepsis group and other groups than in the healthy controls. However, PCT did not differentiate sepsis from infection or SIRS, which may be related to the diagnostic criteria of sepsis. The prognosis of infection varies, including death, remission, sepsis, etc. It may become a link in the progression to sepsis. SIRS standard and infection were used as the diagnostic criteria for sepsis, so there was no statistical significance in the difference analysis of PCT between the sepsis group and the infection group. For IL-6, a prospective, controlled, multicenter study found that IL- 6 can be used as a diagnostic and prognostic biomarker for sepsis and septic shock (21), IL-6 is a better diagnostic indicator of sepsis than PCT and CRP(21). It was also suggested that the diagnostic value of IL-6 in patients with sepsis was almost equal to that of PCT. A meta-analysis suggested that IL- 6 should be used as an adjunctive diagnosis in patients with non-infectious inflammation rather than as a diagnostic indicator(22). There are also studies suggesting that the diagnostic value of PCT is better than IL-6 (AUC59.6, sensitivity $43.6 \%$, specificity $100 \%$ ). In this study, the sepsis group and the healthy control group have the same results (AUC98. $1, \mathrm{P}<0.05)$. Considering that there are 283 mixed infection patients in the sepsis group, it may be related to the patient's severe illness and impaired immune function. However, when sepsis is compared with the other two groups, it shows a certain differential diagnosis significance, which can be used as a tool to identify sepsis, which is conducive to early targeted sepsis bundle therapy to improve prognosis.

In addition, we also found that TBIL was significantly different in sepsis compared with infection or healthy controls, and showed certain diagnostic value. Given that hyperbilirubinemia is a common complication of sepsis, it is used as an indicator of liver function in APA CHEII and sequential organ failure scores (8), which is associated with poor prognosis of sepsis - associated liver injury (25). Some studies also believe that although TBIL is higher in the sepsis group, its correlation is not clinically significant because it is a parameter of the SOPA 
standard(26). Our study did not adopt the SOPA standard, TBIL still showed differences between groups, and comparing the sepsis group and the infection group, the AUC of TBIL was 60.0 , the sensitivity was $55.3 \%$, and the specificity was $64.6 \%$, which is meaningful for differential diagnosis. Therefore, it is believed that it may be beneficial to early diagnosis.

Unlike other sepsis biomarkers such as PCT or CRP, WBC, NE, and D-dimer are the first laboratory test results that clinicians can use. Therefore, we analyzed the diagnostic value of NE, WBC and D-dimer and evaluated their applicability as early diagnosis of sepsis patients. In our study, WBC, IL-6, NE, TBIL showed statistical differences in the comparison between the sepsis group and the infection group. The AUC of NE was 67.6 , the largest of the four, with the highest specificity (95.8\%) but the lowest sensitivity (49\%). The sensitivity and specificity of WBC (AUC66.7) were $51 \%$ and $89.6 \%$, and both NE and WBC had certain value of differential diagnosis, although their performance was general. The study suggested that the leucocyte, NE value increased gradually according to the severity of the infection (27). The diagnostic accuracy of total leukocyte parameters can provide valuable information for the diagnosis and follow-up of sepsis in patients with liver damage in ICU. Neutrophil dysfunction may actively participate in the development of sepsis (27). In our study, both NE and WBC in the sepsis group and the healthy control group showed diagnostic significance, and the diagnostic efficiency was fair (NE: AUC86.5, sensitivity 79.5\%, specificity $100 \%$; WBC: AUC77.9, sensitivity $66.7 \%$, specificity $100 \%$ ). The lack of statistical significance in the comparison between the sepsis group and the SIRS group is related to our use of the Sepsis2.0 diagnostic criteria. prospective cohort study of early biomarkers of sepsis in burn patients: NE can be used as a biomarker for predicting/early diagnosing sepsis. The use of therapeutic interventions for neutrophil dysfunction may reduce the incidence of nosocomial infection and sepsis after burns. The study suggests that the total number of white blood cells $(p<0.05)$ in patients with sepsis is higher than that in the control group, which is consistent with our analysis. There is no statistical difference in platelets between the control group and sepsis patients, which may be related to the impact of their choice of lung disease as the control group on the results $(28,29)$. In our data analysis, although PLT is statistically different in the comparison between the sepsis group and the healthy control group $(P<0.05)$, it has no diagnostic value. In the comparison between the sepsis group and the SIRS group reflect a certain significance of differential diagnosis and can be used as an auxiliary indicator. A study of ICU patients with liver disease showed that it was related to significant differences in WBC and CRP. The diagnostic accuracy of white blood cell parameters may provide valuable information for the diagnosis and follow-up of sepsis in ICU patients (especially patients with liver dysfunction) (27). There are also many studies suggesting that the combination of white blood cell count or neutrophil count with other indicators may increase the detection rate of sepsis, which can guide us in the next step of research (14).

D-dimer is a fibrin degradation product, and the increase of D-dimer level indicates the presence of hypercoagulable state and secondary fibrinolysis in the body. Many factors can increase it, such as infection, DIC, heart or kidney damage, thrombolytic therapy, etc. Sepsis is a clinical syndrome that complicates severe infections. It is characterized by the main manifestations of inflammation (vasodilation, white blood cell accumulation, increased vascular permeability, etc.) appearing in tissues far away from the infection site (30). Inflammation and clotting affect each other, Studies have shown that clotting can be activated by inflammation, which leads to endothelial damage and the formation of exudative platelet aggregation (28). In our study, D-dimer showed excellent diagnostic value in the sepsis group, and we believed that D-dimer, PCT and CRP were all conducive to the early detection of sepsis. In one study of mice(28), a sepsis model was created by intraperitoneal injection of liposolysaccharide (LPS). 24 hours later, there was an exudative platelet aggregation in the liver, and the levels of AST, ALT, and DBIL were significantly elevated. This study suggests that Neutrophil Extracellular Traps (NETs) formation and platelet aggregation are the first steps in the development of liver dysfunction in sepsis. Inflammation and coagulation play a key role in the pathogenesis of septicemia, leading to multi-organ failure, echoing Sepsis3.0 diagnostic criteria.

\section{Conclusion}

Infection is a link in the progression to sepsis, but the treatment regimen for infection and sepsis is different. Early identification of sepsis and early implementation of targeted treatment are beneficial for patients with sepsis. In this stud, traditional inflammatory markers CRP,IL- 6 and PCT show diagnostic value, among which PCT shows the best performance when compared with control group, but only IL-6 shows certain differential diagnostic significance. In early biochemical markers, NE, WBC, and D-dimers have been found to aid in the early diagnosis of sepsis, and D-dimers perform best in this group. This has certain clinical practice value for early diagnosis of sepsis and is conducive to timely medical intervention for patients with sepsis, WBC and NE may have differential diagnostic significance in the sepsis and infection groups, and PLT may have differential diagnostic significance in the sepsis and SIRS groups. Next, we can include more patients for prospective study to further determine their diagnostic significance.

\section{Abbreviations}

NE, neutrophil count ; WBC , white blood cell count ; PCT, procalcitonin; CRP, C-reactive protein; IL-6, interleukin-6; TBIL, total bilirubin ; MCV, mean corpuscular volume; MO, Monocyte count; PLT, blood platelet; HB, hemoglobin; PT, prothrombin time; ALB, albumin; Cr, creatinine; BNP, Btype natriuretic peptide; LAC, Lactic acid; SIRS, systemic inflammatory response syndrome; AUC, area under the curve; 


\section{Declarations}

\section{Funding}

The present study was supported by the National Natural Science Foundation of China (grant no. 81660353).

\section{Ethical Approval}

\#A: The authors are accountable for all aspects of the work in ensuring that questions related to the accuracy or integrity of any part of the work are appropriately investigated and resolved. \#B: The authors are accountable for all aspects of the work in ensuring that questions related to the accuracy or integrity of any part of the work are appropriately investigated and resolved. All procedures performed in studies involving human participants were in accordance with the ethical standards of the institutional and/or national research committee(s) and with the Helsinki Declaration (as revised in 2013). Written informed consent was obtained from the patient.

\section{Consent to Participate}

Informed consent was obtained from all individual participants included in the study.

\section{Consent to Publish}

The participant has consented to the submission of the case report to the journal.

\section{Authors Contributions}

(I) Conception and design: Yan Meng, Yue Wang, Wenbin Qiao, Fengming Tian, Tao Zhang, Xiumin Ma; (II) Administrative support: Xiumin Ma, Tao Zhang; (III) Provision of study materials or patients: Yue Wang, Yumei Liu, Liang Wang, Jiahui Fan, Xuanzheng Wang, Tao Zhang; (IV) Collection and assembly of data: Yue Wang, Jiahui Fan, Xuanzheng Wang; (V) Data analysis and interpretation: Yue Wang, Jiahui Fan, Xuanzheng Wang; $(\mathrm{VI})$ Manuscript writing: All authors; (VII) Final approval of manuscript: All authors.

\section{Competing Interests}

The authors have no conflicts of interest to declare that are relevant to the content of this article.

\section{Availability of data and materials}

All data reported in this study are included in this published article. Further details can be available from the corresponding author on request.

\section{References}

1. Takada T, Hoogland J, Yano T, et al. Added value of inflammatory markers to vital signs to predict mortality in patients suspected of severe infection. Am J Emerg Med. 2020; doi:10.1016/j.ajem.2019.11.030

2. Napolitano LM. Sepsis 2018: Definitions and Guideline Changes. Surg Infect (Larchmt). 2018; doi:10.1089/sur.2017.278

3. Fernando SM, Reardon PM, Rochwerg B, et al. Sepsis-3 Septic Shock Criteria and Associated Mortality Among Infected Hospitalized Patients Assessed by a Rapid Response Team. Chest. 2018; doi:10.1016/j.chest.2018.05.004

4. Rudd KE, Johnson SC, Agesa KM, et al. Global, regional, and national sepsis incidence and mortality, 1990-2017: analysis for the Global Burden of Disease Study. The Lancet. 2020; doi:10.1016/s0140-6736(19)32989-7

5. Iba T, Ito T, Maruyama I, et al. Potential diagnostic markers for disseminated intravascular coagulation of sepsis. Blood Rev. 2016; doi:10.1016/j.blre.2015.10.002

6. Patel P, Walborn A, Rondina M, et al. Markers of Inflammation and Infection in Sepsis and Disseminated Intravascular Coagulation. Clin Appl Thromb Hemost. 2019; doi:10.1177/1076029619843338

7. Singer M, Deutschman CS, Seymour CW, et al. The Third International Consensus Definitions for Sepsis and Septic Shock (Sepsis-3). JAMA. 2016; doi:10.1001/jama.2016.0287

8. Husabo G, Nilsen RM, Flaatten H, et al. Early diagnosis of sepsis in emergency departments, time to treatment, and association with mortality: An observational study. PLoS One. 2020; doi:10.1371/journal.pone.0227652

9. Ferrer R, Martin-Loeches I, Phillips G, et al. Empiric antibiotic treatment reduces mortality in severe sepsis and septic shock from the first hour: results from a guideline-based performance improvement program. Crit Care Med. 2014; doi:10.1097/ccm.0000000000000330

10. Churpek MM, Snyder A, Han X, et al. Quick Sepsis-related Organ Failure Assessment, Systemic Inflammatory Response Syndrome, and Early Warning Scores for Detecting Clinical Deterioration in Infected Patients outside the Intensive Care Unit. Am J Respir Crit Care Med. 
2017; doi:10.1164/rccm.201604-08540C

11. Angus DC, van der Poll T. Severe sepsis and septic shock. N Engl J Med. 2013; doi:10.1056/NEJMra1208623

12. Font MD, Thyagarajan B, Khanna AK. Sepsis and Septic Shock - Basics of diagnosis, pathophysiology and clinical decision making. Med Clin North Am. 2020; doi:10.1016/j.mcna.2020.02.011

13. Hamed S, Behnes M, Pauly D, et al. Diagnostic value of Pentraxin-3 in patients with sepsis and septic shock in accordance with latest sepsis-3 definitions. BMC Infect Dis. 2017; doi:10.1186/s12879-017-2606-3

14. Crouser ED, Parrillo JE, Seymour C, et al. Improved Early Detection of Sepsis in the ED With a Novel Monocyte Distribution Width Biomarker. Chest. 2017; doi:10.1016/j.chest.2017.05.039

15. Ljungstrom L, Pernestig AK, Jacobsson G, et al. Diagnostic accuracy of procalcitonin, neutrophil-lymphocyte count ratio, C-reactive protein, and lactate in patients with suspected bacterial sepsis. PLoS One. 2017; doi:10.1371/journal.pone.0181704

16. Vincent JL, Martin GS, Levy MM. qSOFA does not replace SIRS in the definition of sepsis. Crit Care. 2016; doi:10.1186/s13054-016-1389-Z

17. Disselkamp M, Coz Yataco AO, Simpson SQ. POINT: Should Broad-Spectrum Antibiotics Be Routinely Administered to All Patients With Sepsis as Soon as Possible? Yes. Chest. 2019; doi:10.1016/j.chest.2019.05.030

18. Seymour CW, Gesten F, Prescott HC, et al. Time to Treatment and Mortality during Mandated Emergency Care for Sepsis. N Engl J Med. 2017; doi:10.1056/NEJMoa1703058

19. Caraballo C, Jaimes F. Organ Dysfunction in Sepsis: An Ominous Trajectory From Infection To Death. Yale J Biol Med. 2019;

20. Jamsa J, Ala-Kokko T, Huotari V, et al. Neutrophil CD64, C-reactive protein, and procalcitonin in the identification of sepsis in the ICU - Posttest probabilities. J Crit Care. 2018; doi:10.1016/j.jcrc.2017.08.038

21. Song J, Park DW, Moon S, et al. Diagnostic and prognostic value of interleukin-6, pentraxin 3, and procalcitonin levels among sepsis and septic shock patients: a prospective controlled study according to the Sepsis-3 definitions. BMC Infect Dis. 2019; doi:10.1186/s12879-0194618-7

22. Takahashi W, Nakada TA, Yazaki M, et al. Interleukin-6 Levels Act as a Diagnostic Marker for Infection and a Prognostic Marker in Patients with Organ Dysfunction in Intensive Care Units. Shock. 2016; doi:10.1097/SHK.0000000000000616

23. Jämsä J, Ala-Kokko T, Huotari V, et al. Neutrophil CD64, C-reactive protein, and procalcitonin in the identification of sepsis in the ICU - Posttest probabilities. J Crit Care. 2018; doi:10.1016/j.jcrc.2017.08.038

24. Wacker C, Prkno A, Brunkhorst FM, et al. Procalcitonin as a diagnostic marker for sepsis: a systematic review and meta-analysis. Lancet Infect Dis. 2013; doi:10.1016/s1473-3099(12)70323-7

25. Cui Y, Shan Y, Chen R, et al. Elevated Serum Total Bilirubin Level Is Associated with Poor Outcomes in Pediatric Patients with SepsisAssociated Liver Injury. Can J Infect Dis Med Microbiol. 2018; doi:10.1155/2018/4591729

26. Tutak E, Ozer AB, Demirel I, et al. The relationship between serum bilirubin level with interleukin-6, interleukin-10 and mortality scores in patients with sepsis. Niger J Clin Pract. 2014; doi:10.4103/1119-3077.134057

27. Buoro S, Mecca T, Azzara G, et al. Extended leukocyte differential count and C-reactive protein in septic patients with liver impairment: diagnostic approach to evaluate sepsis in intensive care unit. Ann Transl Med. 2015; doi:10.3978/j.issn.2305-5839.2015.09.41

28. Sakurai K, Miyashita T, Okazaki M, et al. Role for Neutrophil Extracellular Traps (NETs) and Platelet Aggregation in Early Sepsis-induced Hepatic Dysfunction. In Vivo. 2017; doi:10.21873/invivo.11169

29. Hampson P, Dinsdale RJ, Wearn CM, et al. Neutrophil Dysfunction, Immature Granulocytes, and Cell-free DNA are Early Biomarkers of Sepsis in Burn-injured Patients: A Prospective Observational Cohort Study. Ann Surg. 2017; doi:10.1097/SLA.0000000000001807

30. Westerdijk K, Simons KS, Zegers M, et al. The value of the neutrophil-lymphocyte count ratio in the diagnosis of sepsis in patients admitted to the Intensive Care Unit: A retrospective cohort study. PLoS One. 2019; doi:10.1371/journal.pone.0212861

\section{Figures}


A
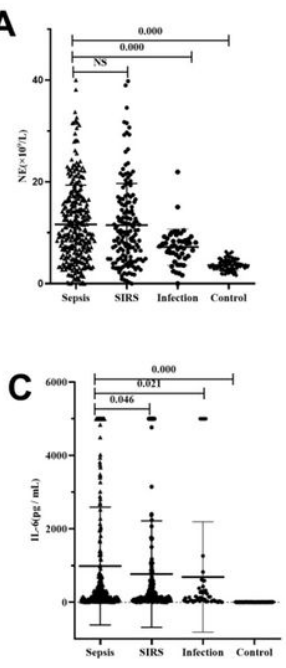

E
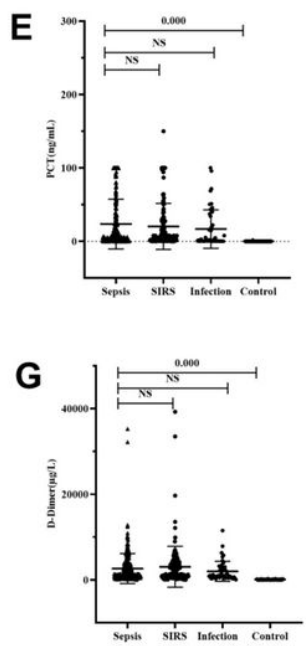

B

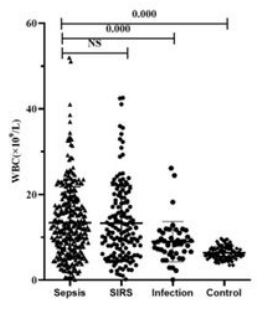

D

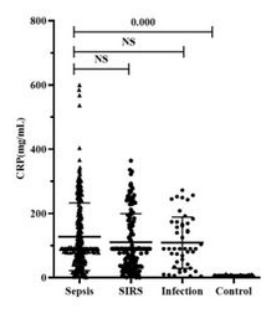

$\mathbf{F}$
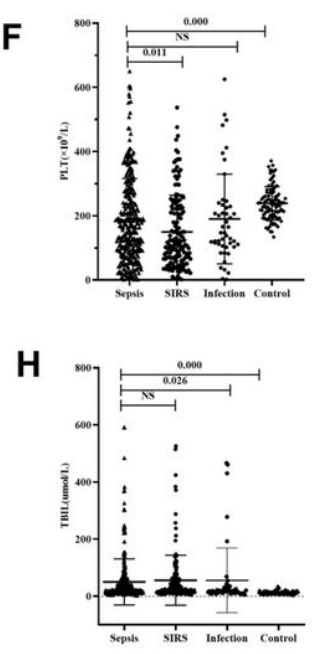

\section{Figure 1}

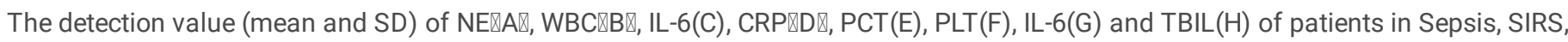
Infection and Healthy controls (significances between groups were calculated with Kruskal-WallisH analyses). 


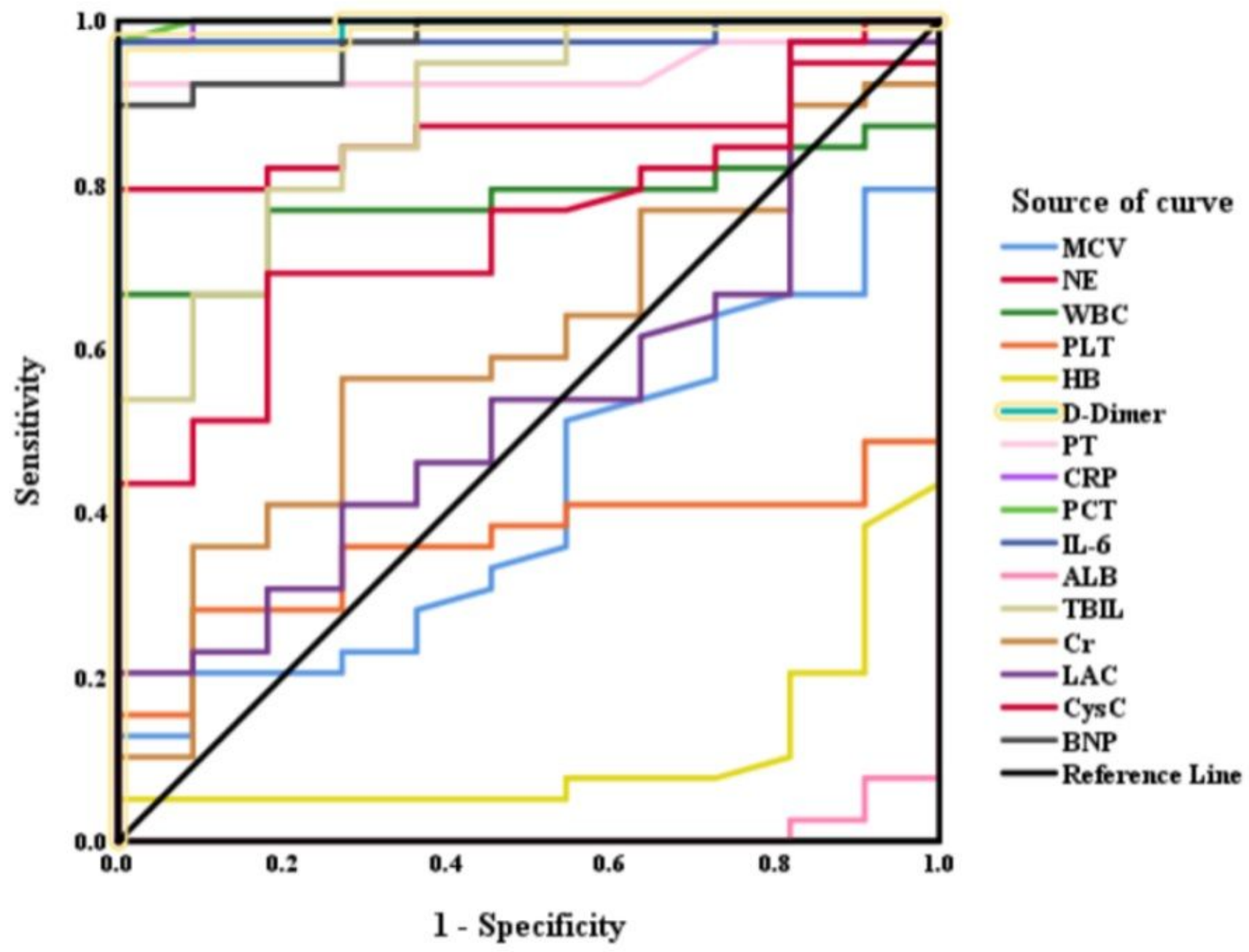

Figure 2

ROC curves (with AUCs) comparing Laboratory test results [MO, NE, WBC, PLT, HB, D-Dimer, PT, CRP, PCT, IL-6, ALB, TBIL, Cr, LAC, CysC and BNP] discriminating between Sepsis Group and Control Group. 


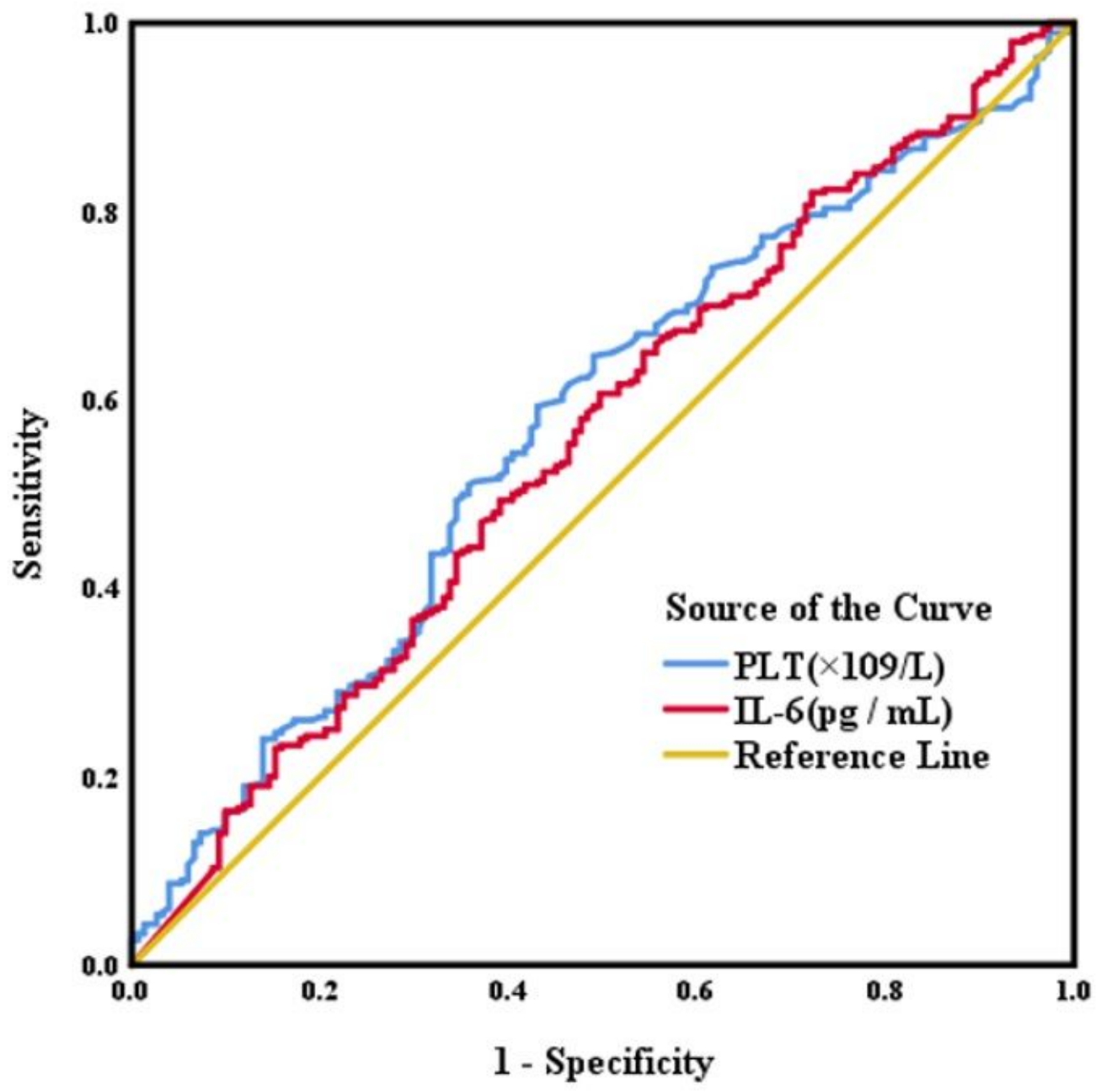

Figure 3

ROC curves (with AUCs) comparing biomarkers [PLT, IL-6] discriminating between Sepsis Group and SIRS Group. 


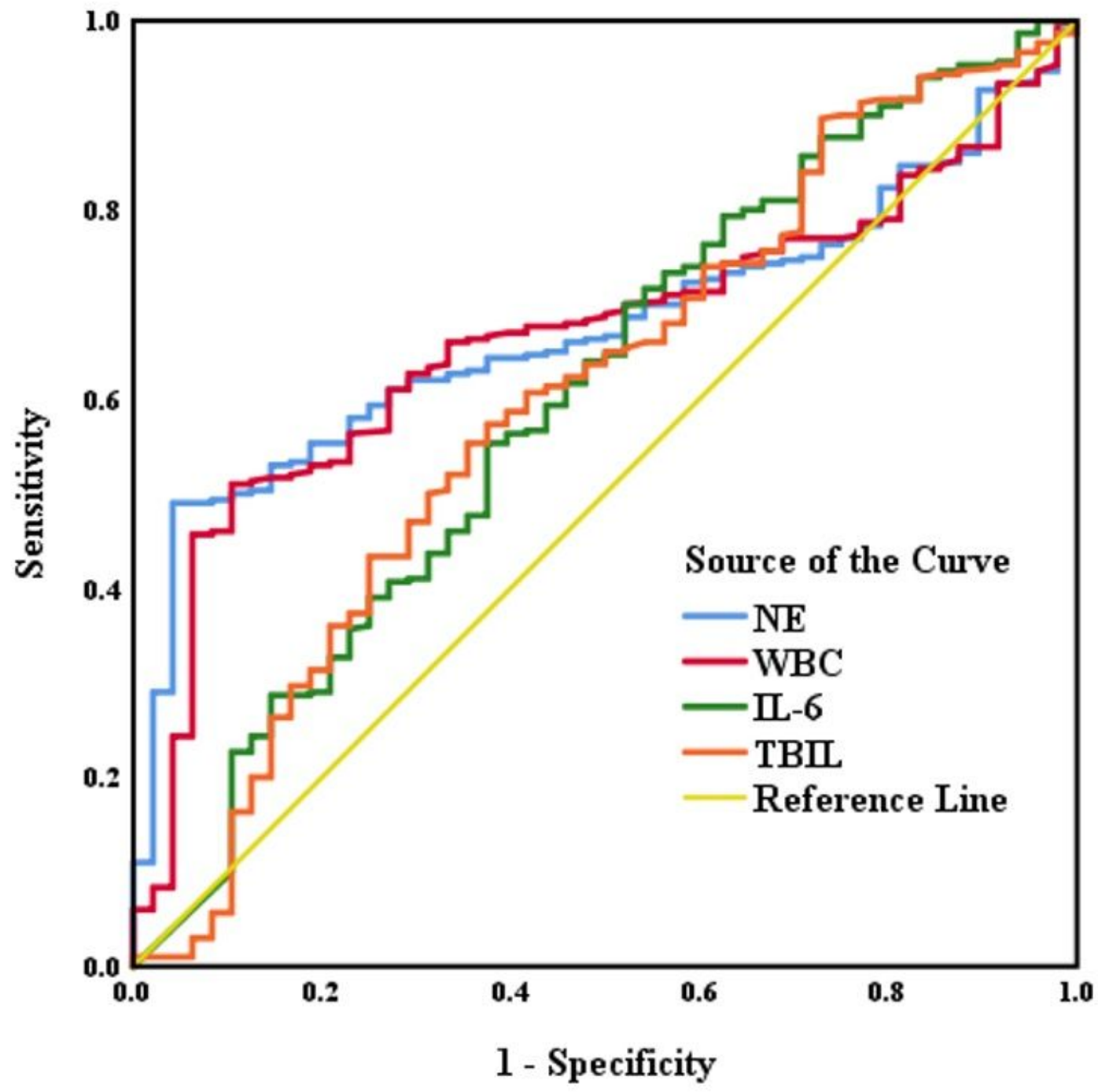

Figure 4

ROC curves (with AUCs) comparing biomarkers [NE, WBC, IL-6, TBIL] discriminating between Sepsis Group and Infection Group. 